Magna Scientia Advanced Research and Reviews

eISSN: 2582-9394

Cross Ref DOI: $10.30574 / \mathrm{msarr}$

Journal homepage: https://magnascientiapub.com/journals/msarr/

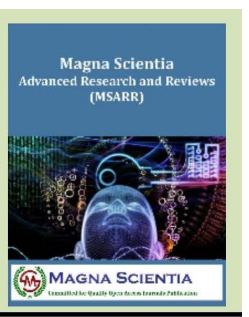

(REVIEW ARTICLE)

\title{
Effects of cystic fibrosis on the oral cavity
}

\author{
H. Goumghar * and M. Sidqui \\ Faculty of Dental Medicine of Casablanca, Hassan II University, Casablanca, Morocco.
}

Magna Scientia Advanced Research and Reviews, 2021, 03(02), 061-072

Publication history: Received on 10 November 2021; revised on 13 December 2021; accepted on 15 December 2021

Article DOI: https://doi.org/10.30574/msarr.2021.3.2.0089

\begin{abstract}
Cystic fibrosis is a complex, lethal, multi-system autosomal recessive disease resulting from mutations on chromosome 7 that cause dysfunction of an ion channel located on epithelial surfaces. Pulmonary disease is the leading cause of morbidity and mortality in CF. The specific dental manifestations of the disease may result from the condition itself or from complications of treatment. This study presents the dental problems observed in patients with cystic fibrosis and reported in the literature. Particular attention was paid to dental caries, disturbances in the mineralization of hard dental tissues, gingivitis and changes in the content and properties of saliva.
\end{abstract}

Keywords: Cystic Fibrosis; Oral health; Dental Caries; Dental Enamel Hypoplasia; Periodontal Diseases; Saliva.

\section{Context}

Cystic fibrosis is a complex disease that requires careful multidisciplinary management. It is imperative that the dental surgeon be aware of the disease processes, systemic and dental manifestations related either to the disease itself or as a result of treatment [1]. The effect of cystic fibrosis on the oral cavity has been of interest to researchers for many years, but has resulted in contrasting findings [2]; particular attention has been paid to disturbances in the mineralization of hard dental tissues, dental caries, periodontal disease, and changes in the composition and properties of saliva.

\section{Enamel defects and dental hard tissue}

The effects on the tooth germ in the so-called pre-eruptive phase result in enamel defects [3]. This term describes a series of enamel appearances whose structure is disturbed during its developmental stages [4]. The wide variety of factors that can affect the developmental process of dental hard tissues means that enamel and dentin defects can present a wide range of clinical features [5]: Disorders in the early stages of enamel development are suggestive of enamel hypoplasia [4], which is a quantitative defect involving the partial or total absence of enamel, it presents as pits, hollows or grooves [6]. On the other hand, disorders occurring during the calcification or maturation stage can cause hypomineralization [4], which is a qualitative defect characterized by a white or discolored area with a smooth surface and normal enamel thickness [6].

Hypomineralization of molar incisors (MIH) is a special form of the described defect. It is defined as a dysfunction in enamel development that affects one or more molars with frequent co-existing lesions in the incisors. Clinically, enamel opacities of various sizes and colors are observed, the enamel is soft and porous and tends to be easily destroyed [7].

Liver disease associated with cystic fibrosis may result in hyperbilirubinemia due to the breakdown products of hemolysis. This can cause green staining of enamel, dentin, and dental soft tissues (Fig. 1) [8].It has been reported that children with cystic fibrosis have coronal staining of permanent teeth. These findings appear to be historical, related to

\footnotetext{
${ }^{*}$ Corresponding author: H Goumghar

Faculty of Dental Medicine of Casablanca, Hassan II University, Casablanca, Morocco.

Copyright $(2021$ Author(s) retain the copyright of this article. This article is published under the terms of the Creative Commons Attribution Liscense 4.0.
} 
the use of tetracycline antibiotics during dental development [1]. Recognition of this side effect has led to the adoption of alternative antibiotic prescribing for all children, including those with $\mathrm{CF}$, thereby reducing the risk of hard dental tissue discoloration [9]. Other antibiotics, including Doxycycline and Linezolid, have also been associated with tooth discoloration and may be used in CF. Nebulized antibiotics, particularly off-label use of the antibiotic Carbapenem Meropenem, have been associated with marked black discoloration of teeth (Fig. 2) [1].

\subsection{Etiologies of enamel defects}

The factors associated with defects in tooth enamel development are numerous and often unknown [10]. In patients with cystic fibrosis, clinical observations indicate enamel defects with varying degrees of abnormalities [3]. Regulation of $\mathrm{pH}$ has been shown to be critical for apatite deposition, crystallite growth, proteinase optimization, and ameloblast function in the developing enamel [5]. Mutations in the CFTR gene cause altered pH during enamel development, resulting in lack of calcium influx during enamel maturation, hypomineralization, and impairment of normal crystallite growth and protein processing functions required for optimal enamel formation [11].

Enamel defects in patients with CF may also be a side effect of systemic antibiotic therapy for pulmonary exacerbations as well as diseases such as infection or malnutrition resulting from digestive system disorders [3]. Studies suggest that multiple surgical procedures on a child in the early years may have adverse effects on tooth development [12]. The development of opacities may also be related to other conditions: for example, prematurity, low birth weight, ingestion of fluoride from toothpaste, and disorders such as celiac disease and asthma [13].

It has been shown that cystic fibrosis may be associated with delayed tooth eruption [14]. It is thought that dental germ development, because of its high sensitivity to systemic disturbances (CFTR dysfunction, systemic conditions, medications used), often acts as a biomarker of systemic involvement [3].

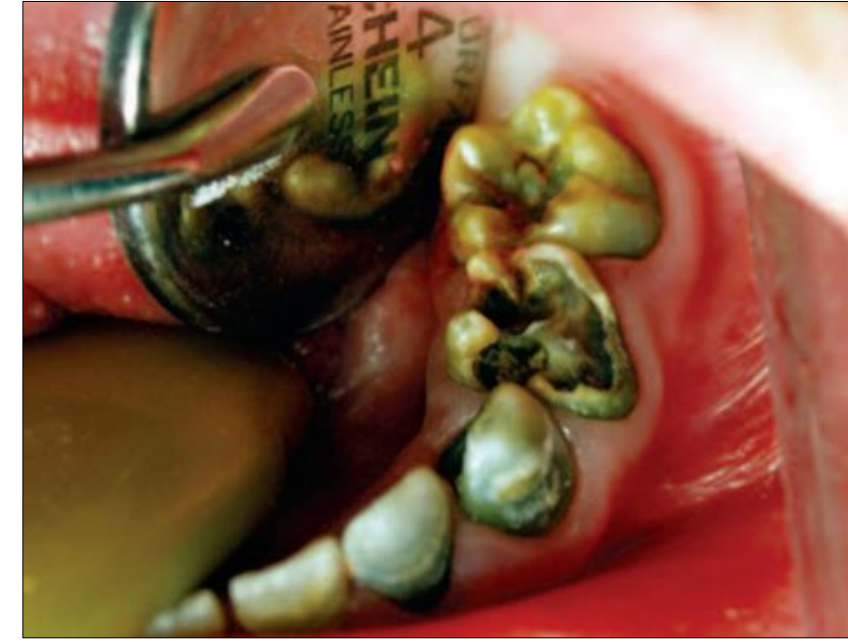

Figure 1 Clinical picture of primary maxillary left mandibular molars showing hyperbiliruminemia stain affecting enamel and dentin, caries and enamel hypoplasia [8].

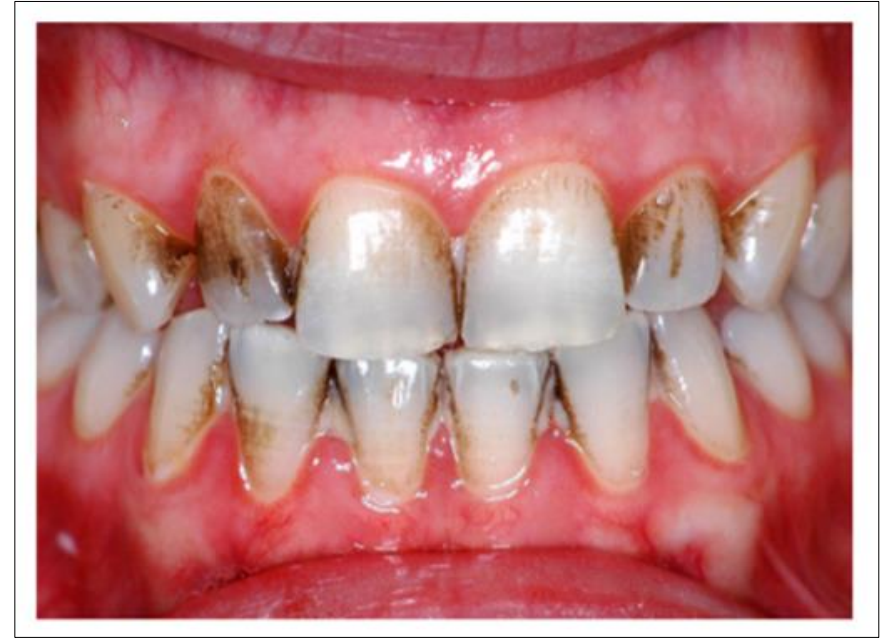

Figure 2 Black discolouration of teeth following the utilisation of the carbapenem antibiotic meropenem [1]

\subsection{Prevalence of dental hard tissue defects in patients with CF}

A number of studies have reported enamel defects associated with cystic fibrosis in humans: Abu-Zahra and colleagues reported that many patients with CF have a higher prevalence of enamel defects compared to their healthy counterparts [12]; The same observation was confirmed by other investigators : Pawlaczyk-Kamieńska and colleagues who also added that the defects were more severe, and more teeth were affected in CF patients [15]; Similar data were obtained by Narang et al. who compared a group of patients with CF with patients with other diseases of the respiratory system [16].

Regarding the most frequently observed dysfunction: studies have found it to be enamel hypoplasia [17] [10]; while others have determined that enamel opacities were the most frequent type of defect [6] [16]. 
After comparing children with CF with healthy children, studies agree that there was little difference in the primary dentition [14], with statistical significance mostly in the permanent teeth [7], more frequently in the permanent first molars and maxillary central incisors [6]. Teeth that are formed during the embryonic period are less exposed to deterioration factors [3];

This explains the minimal effect on primary teeth when compared with permanent teeth, which have a higher percentage of enamel alterations [18].

Children with cystic fibrosis have a high incidence of tooth discoloration when systemic tetracyclines are taken during tooth formation. With the advent of alternative antibiotics, the incidence of tooth discoloration is decreasing [9].

Enamel defects are of concern because on the one hand they can lead to aesthetic problems [12], and on the other hand the reduction in enamel hardness and the increase in porosity promote the penetration of pathogenic bacteria into the tissues and the development of carious lesions. It is therefore necessary to implement early dental care and to develop an individual program of long-term prophylaxis and treatment to prevent complications such as pulpitis and periapical infections, which could be a source of self-infection for patients with cystic fibrosis [15].

\section{Dental caries}

The etiology of the most common oral diseases (dental caries, periodontal disease) is multifactorial. Health conditions, drug therapy, salivary profile, socioeconomic factors and habits are important in the initiation and progression of these diseases [3].

\subsection{Factors influencing caries initiation and progression in patients with $\mathbf{C F}$}

Theoretically, patients with cystic fibrosis could be considered as patients at high risk for caries [2]. Due to the thickening of mucus, nutrient absorption from food is reduced, resulting in pancreatic insufficiency [19] requiring a special diet and constant supplementation with pancreatic enzymes. The daily caloric requirements for adequate weight maintenance in CF are 130-150\% of the regular requirements of healthy adults. These requirements are met by frequent consumption of high-energy meals and snacks, such as confectionery, sweetened beverages and baked goods [7]. To reduce the risk of caries, nutritional needs can be met by offering nutrient-dense foods, such as those rich in fats and oils, instead of foods high in sugar.

This can be accomplished by adding butter to foods such as soups, adding creamy rich sauces to meals, and encouraging the child to eat cheese with crackers as a snack [14].

Frequent use of supplements containing glucose polymers that break down in the oral cavity into monosaccharides is also recommended. These supplements are typically used as powders added to dishes or as high-sugar drinks [7]. The use of aerosols with mucolytic and expectorant properties, the increased use of specific antibiotics, and the correct use of dietary supplements and pancreatic enzymes have allowed cystic fibrosis patients to survive longer. Unfortunately, the acidity of the aerosols, combined with the carrier (lactose or other sugar), may play an important role in the development of caries [20]. People with cystic fibrosis have to take different treatments several times a day, frequently taking medication in the form of syrups or sweetened suspensions for long periods of time [7]; oral antibiotics, for example, are usually dosed in sweetened suspensions to modify the taste [19]. This explains the presence of carious lesions, especially in the upper temporary teeth [7].

In addition, many other factors may put patients with cystic fibrosis at high risk for dental caries: [21] [1] [14] [18] [2] [9]

- The increase in intraoral levels of Streptococcus mutans;

- High prevalence of gastroesophageal reflux disease;

- High prevalence of enamel alterations.

- Oral breathing caused by chronic nasal and sinus obstructions;

- Irregularity of dental examinations,

- Insufficient use or even refusal of topical fluoride.

- The composition and properties of saliva are also considered; however, reports on this issue are often inconclusive. 
Special attention is also given to parents and caregivers who often tend to overprotect and spoil their children, allowing them too frequent consumption of sweets [7] with the medical concerns that a child with a chronic disease faces, oral health may be perceived as being of lesser importance; thus, the implementation of good dietary and oral hygiene practices may not occur, resulting in an increased risk of caries and gingivitis [14].

\subsection{The prevalence of caries in patients with $\mathrm{CF}$}

Clinically and despite the important risk factors, the prevalence of dental caries appears to be lower in children with cystic fibrosis compared to healthy controls, as pointed out by many authors [12] [22] [23] [21] [24] [7] [25] [26] [9]. Other studies show a higher prevalence of dental caries in patients with CF [27], or no significant difference from the control group [28] [16] [20]. These contrasting results make it difficult to draw conclusions to help understand the nature and extent of oral health problems in patients with cystic fibrosis.

From a practice perspective, it is important to assess the risks of each patient individually, taking into account the broader determinants of health and the status and treatment plan accordingly, ensuring that oral health promotion, prevention, and preventive care are managed appropriately [2].

The low prevalence of caries in children with CF may have several possible explanations [9]: The first is related to improved oral health care, and prevention and education programs for patients and their parents about oral health conditions [12] [3]. The second is based on studies that suggest that pancreatic enzyme supplementation may result in reduced plaque levels and a reduced risk of caries [1] [16].

Third, work has reported that average $\mathrm{pH}$ values are significantly higher, the concentration of calcium and phosphorus ions in saliva is higher, and the buffering capacity is greater, which manifests itself as a positive impact on maintaining the acid-base balance of the oral cavity environment, and promotes remineralization of the teeth.

The increased capacity of salivary buffering systems may be the result of pancreatic enzyme supplementation administered to cystic fibrosis patients [7]. A potential explanation is based on previous work indicating that protection against caries is associated with stimulated saliva, rather than unstimulated saliva, because stimulated saliva contains a higher mineral content and results in greater salivary buffering capacity and clearance [29].

Protection against the development and progression of dental caries in cystic fibrosis patients is explained by another factor: long-term use of oral antibiotics, which may affect the change in the genotype of the oral microflora [3]. Cystic fibrosis patients use antibiotics to remedy chronic lung infection [30]; the dominant pathogen of the respiratory system is Staphylococcus aureus, the infections it causes are treated with penicillin, which is also effective against mutans streptococci [7], it penetrates the dental biofilm, with good absorption into the periodontal tissue, and can be kept in the periodontal pocket for up to 14 days [30]; this reduces the titer of cariogenic agents in the oral cavity [7].

Data from the literature suggest that adolescents with cystic fibrosis have a higher prevalence of caries than their peers without CF [29] [31] [25]. Due to changes in oral health behaviors such as decreased tooth brushing frequency and poor diet [31]. Studies have shown that there is also another explanation: Frequent antibiotic use appears to protect patients from caries until the mid-teens [32]; After the 10th year of life, the respiratory microbiological profile changes, with infections caused by Pseudomonas aeruginosa becoming more dominant [7], often resulting in a change in antibiotic prescription from Penicillin-based therapy to inhaled Tobramycin, which targets Pseudomonas aeruginosa but does not affect cariogenic bacteria [21].

In fact, taking this gram-negative antibiotic by inhalation can create intraoral ecological pressure, which favors grampositive bacteria such as S. mutans [18]. Therefore, adolescents with cystic fibrosis would lose the benefits of caries protection offered in childhood [30]. With regard to dental erosion, it was not statically significant in children with cystic fibrosis or in the control group of healthy patients. However, esophageal reflux and inhaled medications taken by cystic fibrosis patients may have an influence in the presence of dental erosion [20] [18].

\section{Effects on the state of the periodontium}

The homeostasis of the human microbiome plays a key role in maintaining the health status of the human body. Changes in the composition and function of the human microbiome (dysbiosis) are the cause of important inflammatory infectious diseases such as periodontal disease [33]. 


\subsection{Factors influencing the onset and progression of periodontal infection}

The bacterial complex concept, introduced by Socransky and colleagues (1998), is based on the specific plaque hypothesis, which assumes that a specific microbiota is associated with gingivitis or chronic or aggressive periodontitis, and that the pathogenicity of plaque depends on the presence or increased number of specific microorganisms. On the basis of bacterial correlations, all biofilm periopathogens are divided into four groups (complexes). The species of the different complexes are closely related. In most cases, the species of a particular group appear together, or they are all absent together. This interaction and cooperation between the bacteria in the plaque biofilm leads to a form of stabilization in which all species exist in harmony and balance with their environment. This stabilization of the biofilm is called the climax community [34]. Poor oral hygiene contributes to the accumulation of plaque on the supra- and subgingival surface of the teeth, which is directly associated with inflammation of the periodontal tissues [3].

In patients with cystic fibrosis, many risk factors can alter the body's defense mechanisms and significantly determine the severity and clinical signs of the disease, including:

- Saliva quantity and composition: CFTR dysfunction results in abnormal salivary gland activity, with altered salivary calcium and phosphate composition which can affect tartar formation [1] [16].

- $\quad$ Long-term drug therapy: Chronic use of antibiotics, including inhalation, may reduce the number of bacteria and provide some protection against the onset and progression of periodontal disease [1] [3] [25].

- Level of oral hygiene: Oral hygiene habits may be less effective during periods of exacerbation of the underlying disease, which may increase the risk of periodontal and caries disease (Fig. 3).

- Socioeconomic status should also be considered: The most important indicators are employment status, financial status, and education level [3].

- Cystic fibrosis patients often breathe through the mouth, due to frequent upper respiratory tract infections, which promotes malocclusions and predisposes to periodontitis and oral mucosal inflammation [4].

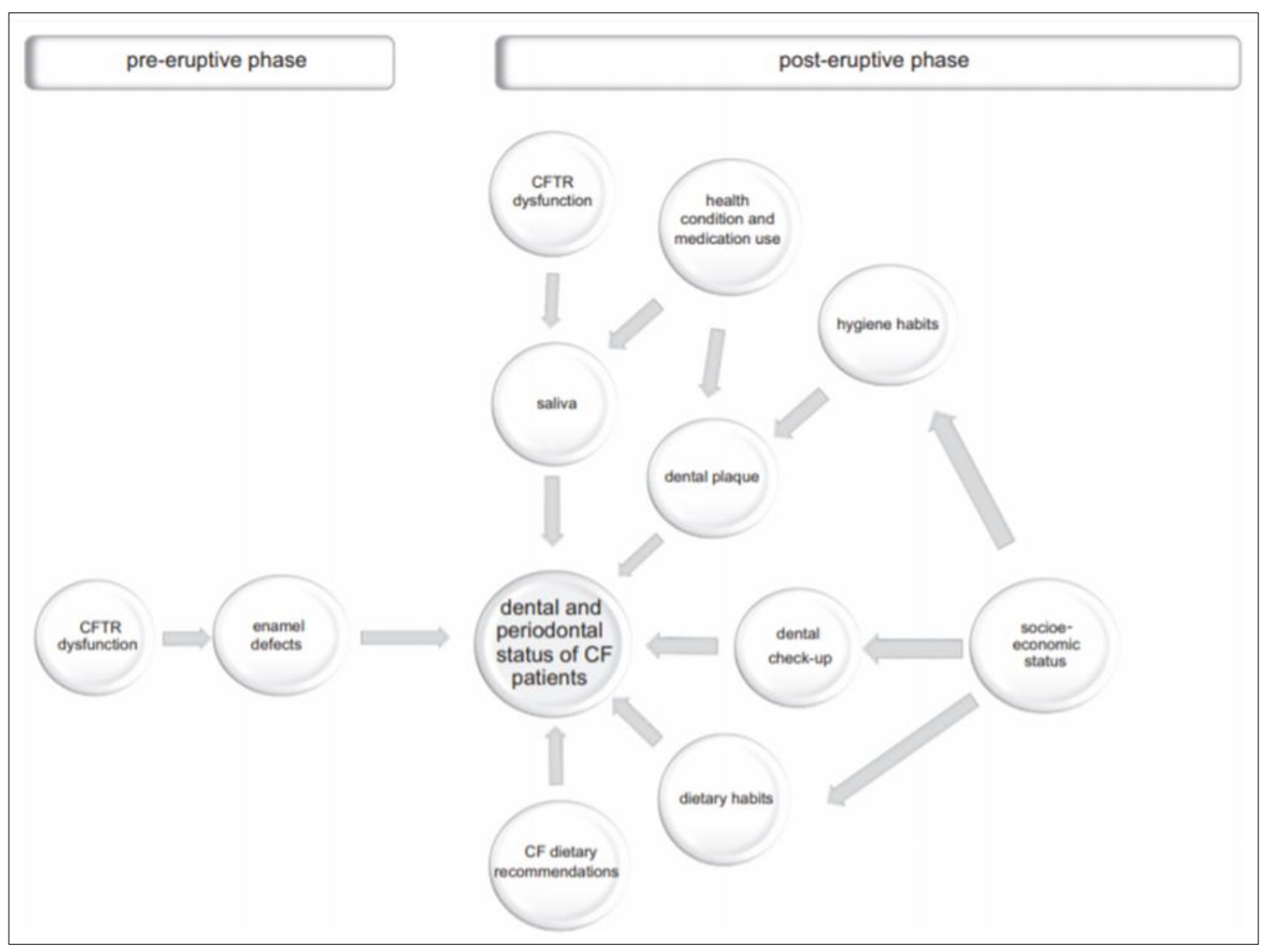

Figure 3 Factors related to dental caries and periodontal disease in CF patients [3]

Among the many factors influencing the onset and progression of periodontal infection are the periopathogens, which are of paramount importance, but their presence in the oral cavity is not always apparent. The clinical picture of periodontal disease depends on the balance of interaction between the microbial biofilm of the dental plaque and the 
host immune response [35]. Most reports indicate a lower risk of gingivitis in patients with cystic fibrosis; however, simultaneously, there is a greater tendency for dental calculus formation [7]

\subsection{Manifestations of cystic fibrosis on the soft tissues of the oral cavity}

Patients with cystic fibrosis are at increased risk for Candida acquisition and colonization due to the use of inhaled steroids, diabetes mellitus, and lifelong antibiotic therapy [1]. Candida species are capable of causing chronic, localized, or systemic infection collectively referred to as "Candidiasis," although they most often act as commensals, but they can sometimes cause opportunistic infections.

Oral thrush usually presents as discomfort associated with dry mouth and associated dysphagia. In some cases, an altered taste is experienced. Diagnosis is usually straightforward and is based on direct observation of white membranous plaques on the oral mucosa or soft palate. This can be confirmed microbiologically by staining a swab or by culturing a rinse of the associated area.

However, despite its frequent isolation from smears, oral and vaginal swabs, the cut-off point for distinguishing between commensalism and colonization remains undetermined. Oral candidiasis is clearly recognized in cystic fibrosis, but the lack of available literature suggests that it is probably misdiagnosed, ignored, or missed in many cases [36].

Oral antibiotics have also been associated with the development of a hairy tongue and its black staining in patients with CF [1]. Although black hairy tongue is usually asymptomatic, patients may occasionally complain of tickling or burning of the tongue, nausea, halitosis, dysgeusia, and an unattractive appearance of the tongue. Black hairy tongue is due to defective scaling of the dorsal surface of the tongue, usually in the posterior third. This defective desquamation prevents normal debridement, resulting in excessive growth and thickening of the filiform papillae, which then collects debris, bacteria, fungi or other foreign material that contributes to the discoloration. The exact mechanism of drug-induced black hairy tongue is currently unknown [37].

There are new treatment modalities for people with cystic fibrosis, including CFTR regulatory modulators, and their use is now widespread. The first of these, Ivacaftor was only introduced in 2012. The vast majority of oral health studies in people with CF were conducted prior to this date. It would be interesting to see what effect, these medications would have on the periodontium [38].

\section{Effects on saliva status}

The oral cavity is constantly exposed to the influence of adverse environmental factors and saliva is the first secretion to come into contact with exogenous substances [39]. It plays an important role in maintaining the health of the oral mucosa and teeth by continuously coating and lubricating the oral tissues [40]. This complex bio-fluid creates a proper ecological balance and provides a barrier against irritants [41]. During cystic fibrosis, there are a series of significant changes in the composition and properties of saliva, which are an expression of salivary gland dysfunction and a side effect of drug therapy [7]. Dry mouth can be a complaint in patients with CF. Saliva tends to be thick mucus with increased lipid content [14].

\subsection{Effects on the salivary glands}

CFTR dysfunction in cystic fibrosis patients affects hydration and mucociliary transport in all exocrine glands, including the salivary glands [29] [13] [11], it makes their respective secretions more viscous than under healthy conditions [4]. Salivary gland hypofunction alters the flow and composition of saliva, which may affect nutritional intake, social interaction, and quality of life [40]. The submandibular gland may be enlarged; secretion from the sublingual gland is thick and tenacious; The parotid gland, which is not a mucus-secreting gland, may have increased flow with high electrolyte content [4].

\subsection{Effects on saliva constituents}

The major constituent of saliva is water, which accounts for $99 \%$ of its composition. The solid components, which are characterized by organic and inorganic molecules, are dissolved in the aqueous medium. Salivary composition varies considerably between individuals and within the same individual under different circumstances; in patients with $\mathrm{CF}$, saliva composition may be altered due to the presence of the CFTR protein in the epithelial cells of the salivary gland excretory ducts [42].

- $\quad$ Effects on organic components 


\subsubsection{Salivary proteins}

A significant increase in salivary protein concentration has been observed in cystic fibrosis patients by several authors [43] [13] [44], this is due to the fact that inflammation induces an increased involvement of specific proteins in this process, while Peker et al. did not establish this correlation [20]. Indeed, salivary proteins are involved in many biological processes, such as maintenance of tissue integrity, oral pH regulation or antibacterial activity. Their levels may depend on circadian rhythm, hormones, mental disorders or oral hygiene. Several authors have hypothesized that there is a regulatory mechanism for salivary protein levels [45].

The second hypothesis that explains the increase in salivary proteins in patients with CF is the fact that it could be associated with an alteration of the integrity of the oral and gingival mucosa, and therefore with an increase in salivary albumins [46]. Authors have confirmed that exacerbation of systemic inflammation in CF leads to increased levels of specific inflammatory proteins in saliva [45].

Sialic acid is a component of glycoproteins, abundantly present in mucin [7]. Sialic acid-rich glycoproteins protect the oral mucosa by lubrication against dryness, friction or rubbing; they also control the permeability and penetration of irritants and bacterial toxins into the mucosa. Impaired glycoprotein formation compromises mucosal integrity and protection [13], and may be one of the causes of bacterial colonization of the airways in patients with CF.

Modesto et al. identified a significant decrease in the concentration of total, free, and combined sialic acid in the saliva of patients with CF compared to healthy subjects [13]. According to these authors, the cause of this phenomenon may lie in the use of mucolytics that, on the one hand, facilitate mucus removal, but on the other hand, may simultaneously interfere with the proper formation of salivary glycoproteins [7].

Changes in protein composition can have dramatic effects on oral health. Thromboplastin, also known as tissue factor or factor III, is an important factor that is not actively found in the blood, but as a component of cell membranes.

It triggers extrinsic blood coagulation with factor VII. The latest reports show a significant decrease in thromboplastin activity in saliva, which may lead to delayed wound healing and a predisposition to intense ble eding resulting from gingival inflammation [7].

In addition, whole saliva samples from young patients with CF were found to have higher levels of protein, antioxidants, and uric acid than those from controls. All of these salivary changes are thought to be related to chronic exposure in the child's oral cavity, oxidative process activity and inflammation. These patients represent biomarkers that may provide further clues to the etiology and monitoring of cystic fibrosis [47].

\subsubsection{Enzymes}

Reduced activity of enzymes with antimicrobial properties, $\alpha$-amylase and salivary peroxidase, could have a negative impact in the oral cavity of patients with $\mathrm{CF}$, as they are more vulnerable to bacterial infections. Amylase is a very abundant salivary protein responsible for the initial digestion of starch, it is also able to bind to bacteria and acquired enamel film [13]. During cystic fibrosis, a significant decrease in salivary $\alpha$-amylase enzymatic activity has been demonstrated; this may have an impact on the caries process by increasing the affinity for cariogenic and periodontopathogen microorganisms [7]

Salivary peroxidase is an antioxidant enzyme that catalyzes the breakdown of hydrogen peroxide from bacterial or leukocyte-derived hydrogen peroxide, thereby reducing oral cellular toxicity. This system belongs to the group of innate defense factors with nonspecific mechanisms of action, including active antibacterial, antiviral and antifungal activity [13].

The major antimicrobial effect of salivary peroxidase systems is mainly bacteriostatic, inhibiting the proliferation of endogenous and exogenous bacterial species in the oral cavity by causing a $\mathrm{pH}$-dependent inhibition of glucose uptake, through the oxidation of the key enzyme of glycolysis, hexokinase. Bacterial sensitivity depends on the bacterial species and the concentrations of hypothiocyanite [48].

Human salivary peroxidase catalyzes the conversion of thiocyanate to hypothiocyanite, which in turn inhibits microbiological growth, with a spectrum covering oral and non-oral Gram-positive and Gram-negative bacteria, viruses and fungi. 
In other tissues such as the airway epithelium, hypothiocyanite generation is decreased in CF due to a defect in CFTRdependent thiocyanate secretion, which leads to the collapse of the oxidative antimicrobial mechanism [13]. The decreased activity of this system observed in saliva from cystic fibrosis patients is likely related to impaired CFTR channel-dependent thiocyanate secretion [7], which may compromise the control of salivary properties of the oral biofilm. This reduction is in agreement with previous studies that have demonstrated an increased susceptibility to oxidative damage resulting from alterations in the antioxidant system [13].

Among the various enzymes, saliva contains cathepsin D, which is one of the cascade proteolytic enzymes. Physiological wear and tear results in the appearance of traces of this enzyme in serum and body fluids, including saliva. According to the study by Minarowska et al, cathepsin D activity in saliva of a cystic fibrosis patient is significantly higher than in healthy controls before stimulation of excretion by kerosene pellets. Cathepsin D may be involved in a prolonged inflammatory response in the lung and excessive apoptosis leading to fibroblast activation, and subsequently the induction of tissue remodeling with pulmonary fibrosis [42].

Studies by Nazaryan et al. showed significant differences in local immunity indices of the oral mucosa and periodontal tissue status in children with cystic fibrosis compared with the control group. Disruption of oral mucosal immunity in children with cystic fibrosis is manifested by a 1.5-fold decrease in lysozyme activity in mixed saliva and a 1.4 -fold decrease in IgA secretory immunoglobulin levels. This results in a 3.7-fold increase in the degree of dysbiosis in the oral cavity [49].

\subsubsection{Mucins}

Mucins provide direct dental protection against caries by aggregation and subsequent elimination of streptococcal strains from the oral cavity; as a major component of the acquired pellicle, they interact with hard dental tissues, thus contributing to the microbiological composition of the mouth [50]. The protective effects of mucins were confirmed by Baughan et al. [51], who showed a correlation between increased salivary titers of S. mutans and reduced mucin levels.

\section{Effect on inorganic ions}

Under the right conditions, salivary secretion is a two-step process: the first step takes place in the acini and the second in the salivary duct. In all salivary ducts, sodium ions are actively reabsorbed, while potassium ions are secreted. Therefore, the concentration of sodium ions in saliva is low, while the concentration of potassium ions is increased [52] [7]. One study [52] showed that the concentration of salivary ions depends on the activity of the CFTR protein expressed in the salivary ducts, and that mutations in CFTR lead to abnormal salivary ion concentrations, acting mainly on chloride and sodium values.

Several studies have found that salivary calcium and phosphorus ion values were significantly higher in the group of patients with CF; this high concentration has a positive impact on maintaining the acid-alkaline balance of their oral cavity environment [7]. It may also explain the higher incidence of stones observed in these patients [47].

According to the available literature on inorganic salivary components in patients with CF, there are increased levels of protein and calcium, which allows for the formation of insoluble calcium-protein complexes that could compromise the function of salivary enzymes [13].

Cystic fibrosis patients have higher salivary levels of chloride, potassium, and sodium ions than healthy individuals [11] [7] [47], this increase is due to the distorted function of the CFTR channel [7]; whereas the trace elements vanadium, chromium, selenium, and arsenic are less present in cystic fibrosis individuals [41].

In addition to an increase in saliva viscoelasticity, there are several differences between healthy and cystic fibrosis subjects in electrolyte and protein concentration. The electrolytes that are increased are calcium, sodium and inorganic phosphate. Thiocyanates seem to be decreased, thus reducing the activity of salivary peroxidases.

With regard to proteins, salivary RNase activity is four times higher in cystic fibrosis than in controls. Due to the increased salivary calcium and protein concentration, insoluble proteocalcic complexes are formed and participate in the formation of intraglandular stones [53]. 


\subsection{Effect on $\mathrm{pH}$ and buffering capacity}

Changes in $\mathrm{pH}$ values and buffering capacity play an important role in maintaining the acid-alkaline balance by regulating the demineralization and remineralization processes of hard dental tissue [7]. The literature is inconsistent regarding salivary $\mathrm{pH}$ and buffering capacity in individuals with $\mathrm{CF}$ [3].

Studies have determined that there is a significant decrease in $\mathrm{pH}$ values compared to healthy controls [45], and a simultaneous decrease in its buffering potential responsible for acid-alkaline balance [11] [7] [20]. However, there is also a study reporting significantly higher mean $\mathrm{pH}$ values and buffering capacity. While the other reports found no significant difference in the above-mentioned parameters [13] [25].

The salivary buffering capacity is largely dependent on bicarbonate and phosphate buffer, as well as salivary proteins. Some authors have shown that CF affects the metabolism of chloride and sodium bicarbonate ions.

According to data in the literature, patients have reduced phosphate buffer activity and unchanged bicarbonate buffer activity [45]. The ability of saliva to buffer acids is essential for maintaining pH values in the oral environment. Bicarbonate ions play a major role in determining the $\mathrm{pH}$ and buffering capacity of saliva that can help protect teeth from attack by bacteria-produced acids [39].

\subsection{Effect on salivary flow}

Salivary flow is an essential parameter that conditions the maintenance of homeostasis of the oral cavity environment [7]. Salivary gland dysfunction refers to a change in saliva composition, dry mouth sensation (or xerostomia) can be a complaint in patients with CF [14], is often associated with salivary gland hypofunction, and can severely affect nutritional intake, social interactions, and quality of life [40]. Although the most recent literature regarding salivary characteristics in patients with CF is inconsistent [3]. Some clinical research has confirmed reduced salivary flow in CF subjects compared to healthy controls [30] [7] [47], while others have shown increased salivary flow in CF individuals; however, other authors have not determined such a dependence [13] [20] [25].

In these studies, the authors concluded that the findings suggest a primary defect related to the disease or a secondary defect due to destruction of the glandular membrane parenchyma [11]. Xerostomia is common in patients with CF, probably due to salivary gland dysfunction and drug effects. Mucosal secretory glands, including submandibular, sublingual, and minor salivary glands, express CFTR protein, resulting in altered salivary gland architecture and function, whereas serous parotid glands do not appear. Xerostomia may be aggravated by mouth breathing due to chronic rhino-sinusitis [1]. Local or systemic disorders and diseases are common causes of compromised salivary secretion. In general, many patients with diseases that affect salivary gland function also undergo treatments that may alter saliva secretion and/or induce xerostomia as an adverse effect. Therefore, it can be difficult to distinguish between what can be attributed to the disease itself and what can be induced by the treatment (e.g., medication).

Thus, a thorough diagnostic workup and early diagnosis of salivary gland dysfunction are essential to provide appropriate evidence-based treatment to prevent oral sequelae and to implement individualized management strategies to alleviate xerostomia [40].

\subsection{The role of salivary biomarkers for diagnosis}

Saliva is being extensively studied for the diagnosis of systemic diseases that affect salivary gland function and saliva composition [41]. It is suggested that it can potentially be used to diagnose cystic fibrosis as an alternative to the sweat test [11] [7]. Saliva, being a useful tool for diagnosis, is a valuable source of clinically relevant information, as its many components act as biomarkers of diseases and systemic conditions of the individual [41].

Saliva could be used for the diagnosis of CF, mainly in cases where it is difficult to obtain the correct amount of sweat and/or screening for the CFTR mutation was difficult and/or reference methods for sweat testing are not available to be implemented or are not easily accessible by the general population [52]. According to the study by yucel and colleagues, children with CF have higher gingival inflammation scores and salivary calprotectin levels that correlate with systemic inflammatory markers. They demonstrated that salivary calprotectin may have armchair diagnostic potential for CF in children [54].

Detection of Pseudomonas aeruginosa in the paranasal sinuses may help prevent or delay bacterial aspiration into the lower airways and chronic lung infection in CF. Nevertheless, paranasal sinus sampling by sinus aspiration (the gold standard method) can be invasive, time-consuming, painful, and costly, and therefore not feasible in the clinical routine 
of most CF centers. Enzyme-linked immunosorbent assay (ELISA) techniques for measurement of IgA-mediated secretory immune response in nasal wash and saliva were tested for diagnostic purposes, the results show an easy, feasible and rapid test that in a short time detected changes in status and risk of exposure to P. aeruginosa in the lower respiratory tract with a two-year lead. The ELISA test for the detection of P. aeruginosa-specific sIgA in saliva appears to be a potential alternative for identifying patients requiring investigation of sinus colonization by P. aeruginosa, which, in turn, could open a window of opportunity to eradicate the bacteria in this compartment before they are aspirated into the lungs [55].

\section{Conclusion}

At the conclusion of this work that we performed, we were able to note a number of findings:

- The higher prevalence and more severe enamel defects in the permanent dentition in patients with cystic fibrosis, compared with healthy individuals, require targeted dental care.

- $\quad$ Children with VD have a low risk of dental caries, this is due to the chronic use of antibiotics also effective against cariogenic bacteria, while adolescents would lose the benefits of the protection against caries offered in childhood, due to the change in the lung biotype. Therefore, it is important to emphasize key oral health messages, especially during the transition from childhood to adolescence.

- The available data indicate a lower risk of gingivitis in patients with cystic fibrosis; however, there is a greater tendency for dental calculus to form.

- $\quad$ Patients with VD are at risk for acquisition of oral candidiasis and black hairy tongue.

- $\quad$ Besides the increased viscoelasticity of saliva, there are several differences between healthy and cystic fibrosis subjects in the composition and properties of saliva, making it an interesting alternative diagnostic tool to sweat testing.

\section{Compliance with ethical standards}

\section{Acknowledgments}

The author would like to thank all the contributors to the realization of this paper.

\section{Disclosure of conflict of interest}

The authors declare no conflicts of interest regarding the publication of this paper.

\section{References}

[1] N Harrington, PJ Barry, SM Barry. « Dental treatment for people with cystic fibrosis », Eur. Arch. Paediatr. Dent. juin 2016; 17(3): 195-203.

[2] E O'Keefe. « Are children and adolescents with cystic fibrosis at lower risk of caries?: Question: What is the prevalence of caries in children with cystic fibrosis (CF)? », Evid. Based Dent. juin 2014; 15(2): 46-47.

[3] T Pawlaczyk-Kamieńska, M Borysewicz-Lewicka, R Śniatała, H Batura-Gabryel, S Cofta. « Dental and periodontal manifestations in patients with cystic fibrosis - A systematic review », J. Cyst. Fibros., p. S1569199318309354. nov. 2018.

[4] N Svsg, R Dasaraju. Dental Concerns of Children with Cystic Fibrosis - An Overview », J. Dent. Orofac. Surg. mai 2016; 01(03).

[5] M Atar, EJ Körperich. « Systemic disorders and their influence on the development of dental hard tissues: A literature review », J. Dent. 2010; 38(4): 296-306.

[6] B Jälevik, A Szigyarto-Matei, A Robertson. « The prevalence of developmental defects of enamel, a prospective cohort study of adolescents in Western Sweden: a Barn I TAnadvarden (BITA, children in dental care) study ", Eur. Arch. Paediatr. Dent. 2018; 19(3): 187-195.

[7] K Herman, M Kowalczyk-Zając, T Pytrus. « Oral cavity health among cystic fibrosis patients: Literature overview », Adv. Clin. Exp. Med. Oct 2017; 26(7): 1147-1153.

[8] E Bimstein, K Magliocca, D Cohen, G Morelli, J Katz. « Hyperbilirubinemic Stain: Location and Extent in Dental Tissues », J. Clin. Pediatr. Dent. Sept 2011; 36(1): 75-78,. 
[9] JA Weddell, BJ Sanders, JE Jones. " Dental Problems of Children with Special Health Care Needs », in McDonald and Avery's Dentistry for the Child and Adolescent, Elsevier. 2016; 513-539.

[10] GF Ferrazzano. "Dental Enamel Defects in Italian Children with Cystic Fibrosis: an observational study », Community Dent. Health. 2012; 29: 106-109.

[11] AC Gonçalves et al. «Saliva as a potential tool for cystic fibrosis diagnosis », Diagn. Pathol. déc. $2013 ; 8$ (1): 46.

[12] R Abu-Zahra, N Antos, T Kump, M Angelopoulou. « Oral health of cystic fibrosis patients at a north american center: A pilot study », Med. Oral Patol. Oral Cirugia Bucal. 2019; e379-e384.

[13] KB da Silva Modesto et al. «Salivary flow rate and biochemical composition analysis in stimulated whole saliva of children with cystic fibrosis », Arch. Oral Biol. 2015; 60(11): 1650-1654.

[14] C Harris, G Smith. « Cystic fibrosis in paediatric dental patients », Dent. Nurs. 2013; 9(8): 464-468.

[15] T Pawlaczyk-Kamieńska, M Borysewicz-Lewicka, R Śniatała, H Batura-Gabryel. « Clinical evaluation of the dental hard tissues in an adult population with cystic fibrosis », Pol. Arch. Intern. Med., juill. 2019.

[16] A Narang. " Oral health and related factors in cystic fibrosis and other chronic respiratory disorders », Arch. Dis. Child. 2003; 88(8): 702-707.

[17] TDPL Azevedo, GCS Feijó, ACB Bezerra. « Presence of Developmental Defects of Enamel in Cystic Fibrosis Patients », déc. 2006.

[18] A Molina-Garcia, L Castellanos-Cosano, G Machuca-Portillo, M Posada-de la Paz. «Impact of rare diseases in oral health », Med. Oral Patol. Oral Cirugia Bucal. 2016.

[19] AA Almuntashiri. « Self-Reported Oral health Assessment and Attitudes Toward Oral Health for Adults With Cystic Fibrosis ». 2018.

[20] S Peker, et al. « with cystic fibrosis in Istanbul, Turkey », Niger. J. Clin. Pract. 2015; 18(1): 5.

[21] DL Chi et al. « Age-related heterogeneity in dental caries and associated risk factors in individuals with cystic fibrosis ages 6-20 years: A pilot study », J. Cyst. Fibros. 2018; 17(6): 747-759.

[22] JKM Aps, J Delanghe, LC Martens. « Salivary Electrolyte Concentrations Are Associated with Cystic Fibrosis Transmembrane Regulator Genotypes », Clin. Chem. Lab. Med. 2002; 40(4):

[23] JKM Aps, GOG Van Maele, LC Martens. «Caries experience and oral cleanliness in cystic fibrosis homozygotes and heterozygotes », Oral Surg. Oral Med. Oral Pathol. Oral Radiol. Endodontology. 2002; 93(5): 560-563.

[24] GF Ferrazzano, S Orlando, G Sangianantoni, T Cantile, A Ingenito. « Dental and periodontal health status in children affected by cystic fibrosis in a southern Italian region », Eur. J. Paediatr. Dent. 10: 4.

[25] S Peker, S Mete, Y Gokdemir, B Karadag, B Kargul. « Related factors of dental caries and molar incisor hypomineralisation in a group of children with cystic fibrosis », Eur. Arch. Paediatr. Dent. 2014; 15(4): $275-280$.

[26] EW Sarvas, CE Huebner, JM Scott, JKM Aps, DL Chi. « Dental utilization for Medicaid-enrolled children with cystic fibrosis: DENTAL UTILIZATION FOR CHILDREN WITH CF », Spec. Care Dentist. 2016; 36(6): 315-320.

[27] Dabrowska, E., et al « Assessment of dental status and oral hygiene in the study population of cystic fibrosis patients in the Podlasie province » Adv Med Sci. 2006;51 Suppl 1:100-3.

[28] LC Martens, JKM. Aps. « Is oral health at risk in people with cystic fibrosis ? », Eur. J. Paediatr. Dent. 7.

[29] AA Alkhateeb, LA Mancl, RB Presland, ML Rothen, DL Chi. « Unstimulated Saliva-Related Caries Risk Factors in Individuals with Cystic Fibrosis: A Cross-Sectional Analysis of Unstimulated Salivary Flow, pH, and Buffering Capacity », Caries Res. 2017; 51(1): 1-6.

[30] Gonçalves, Aline Cristina, et al. « Oral health in patients with cystic fibrosis » J. Oral Sci. 2018, 17, e18160.

[31] DL Chi. « Dental caries prevalence in children and adolescents with cystic fibrosis: a qualitative systematic review and recommendations for future research », Int. J. Paediatr. Dent. 2013; 23(5): 376-386.

[32] JRD Patrick, MA da Fonseca, LM Kaste, S Fadavi, N Shah, H Sroussi. « Oral Health-related quality of life in pediatric patients with cystic fibrosis: ORAL HEALTH QUALITY OF LIFE IN CYSTIC FIBROSIS », Spec. Care Dentist. 2016; 36(4): 187-193. 
[33] S Yost, AE Duran-Pinedo, K Krishnan, J Frias-Lopez. « Potassium is a key signal in host-microbiome dysbiosis in periodontitis ». 25.

[34] AD Haffajee, SS Socransky, MR Patel, X Song. « Microbial complexes in supragingival plaque », Oral Microbiol. Immunol. 2008; 23(3): 196-205.

[35] T Pawlaczyk-Kamieńska, R Śniatała, H Batura-Gabryel, M Borysewicz-Lewicka, S Cofta. « Periodontal Status and Subgingival Biofilms in Cystic Fibrosis Adults », Pol. J. Microbiol. 2019; 68(3): 377-382.

[36] SH Chotirmall, CM Greene, NG McElvaney. « Candida species in cystic fibrosis: A road less travelled », Med. Mycol. 2010; 48(1): S114-S124.

[37] FA Khasawneh, DF Moti, JA Zorek. «Linezolid-induced black hairy tongue: a case report », J. Med. Case Reports. 2013; 7(1): 46.

[38] N Coffey, FO' Leary, F Burke, A Roberts, M Hayes. « Periodontal and oral health status of people with Cystic Fibrosis: a systematic review », J. Dent. 2020; 103: 103509.

[39] S Singh, A Sharma, PB Sood, A Sood, I Zaidi, A Sinha. «Saliva as a prediction tool for dental caries: An in vivo study », J. Oral Biol. Craniofacial Res. 2015; 5(2): 59-64.

[40] SB Jensen, A Vissink, N Firth. « Salivary Gland Disorders and Diseases », Contemp. Oral Med. 2018; 1-85.

[41] ACB da Silva, DR da Silva, SA de M Ferreira, AD de S Rodrigues, A de JR Albuquerque, SA Marinho. « Salivary Diagnostics, Current Reality and Future Prospects », in Emerging Trends in Oral Health Sciences and Dentistry, M. S. Virdi, Éd. InTech. 2015.

[42] A Minarowska. « The activity of cathepsin D in saliva of cystic fibrosis patients ». 4.

[43] JKM Aps. « Oral hygiene habits and oral health in cystic fibrosis », Eur. J. Paediatr. Dent. 7.

[44] G Livnat, L Bentur, E Kuzmisnsky, RM Nagler. « Salivary profile and oxidative stress in children and adolescents with cystic fibrosis », J. Oral Pathol. Med. 2010; 39(1): 16-21.

[45] T Hildebrandt, A Zawilska, A Trzcionka, M Tanasiewicz, H Mazurek, E Świętochowska. " Estimation of Proinflammatory Factors in the Saliva of Adult Patients with Cystic Fibrosis and Dental Caries », Medicina (Mex.). 2020; 56(11): 612.

[46] M Lopes-Pacheco. "CFTR Modulators: Shedding Light on Precision Medicine for Cystic Fibrosis », Front. Pharmacol. 2016; 7.

[47] MA Javaid, AS Ahmed, R Durand, SD Tran. «Saliva as a diagnostic tool for oral and systemic diseases », J. Oral Biol. Craniofacial Res. 2016; 6(1): 67-76.

[48] F Bafort, O Parisi, JP Perraudin, MH Jijakli. « Mode of Action of Lactoperoxidase as Related to Its Antimicrobial Activity: A Review », Enzyme Res. 2014; 1-13.

[49] R Nazaryan, M Tkachenko, N Kovalenko, O Babai, O Karnaukh, V Gargin. " ANALYSIS OF LOCAL IMMUNITY INDICATORS OF THE ORAL CAVITY AND DEGREE OF GINGIVITIS DEPENDING ON MUTATION OF CFTR GENE IN CHILDREN WITH CYSTIC FIBROSIS », Georgian Med. News. 2019; 296: 27-31.

[50] X Gao, S Jiang, D Koh, C.-Y. S. Hsu. « Salivary biomarkers for dental caries », Periodontol. 2000; 70(1): 128-141.

[51] LW Baughan, FJ Robertello, DC Sarrett, PA Denny, PC Denny. «Salivary mucin as related to oral Streptococcus mutans in elderly people: Relationship of mucin and S. mutans », Oral Microbiol. Immunol. 2000; 15(1): 10-14.

[52] AC Gonçalves. et al. « Chloride and sodium ion concentrations in saliva and sweat as a method to diagnose cystic fibrosis », J. Pediatr. (Rio J.). 2019; 95(4): 443-450.

[53] Devoize L., Dallel R. « Salivation » EMC (Elsevier Masson SAS, Paris), Med buc, 28-150-M-10, 2010.

[54] ZPK Yucel, et al. « Salivary biomarkers in the context of gingival inflammation in children with cystic fibrosis », J. Periodontol. 2020; 91(10): 1339-1347.

[55] RM Mauch, et al. "Secretory IgA-mediated immune response in saliva and early detection of Pseudomonas aeruginosa in the lower airways of pediatric cystic fibrosis patients », Med. Microbiol. Immunol. (Berl.). 2019; 208(2): 205-213. 